\title{
Knotted holomorphic discs in $\mathbb{C}^{2}$
}

\author{
By S. Baader at Zürich, F. Kutzschebauch at Bern, and E. F. Wold at Oslo
} in $\mathbb{C}^{2}$.

Abstract. We construct knotted proper holomorphic embeddings of the unit disc

\section{Introduction}

Every classical knot type can be represented by a polynomial embedding of $\mathbb{R}$ in $\mathbb{R}^{3}$ [9]. In particular, there exist topologically distinct polynomial embeddings of $\mathbb{R}$ in $\mathbb{R}^{3}$. Crossing these with another coordinate, we obtain topologically distinct polynomial embeddings of $\mathbb{R}^{2}$ in $\mathbb{R}^{4}$. In contrast, all polynomial embeddings of $\mathbb{C}$ in $\mathbb{C}^{2}$ are topologically equivalent, in fact even connected by a polynomial automorphism of $\mathbb{C}^{2}$. The first complete algebraic proof of this fact is due to Abhyankar and Moh [1]; later a purely knot theoretical proof was found by Rudolph [6]. It is an open question whether proper holomorphic embeddings of the complex plane $\mathbb{C}$ or of the unit disk $\triangle \subset \mathbb{C}$ in $\mathbb{C}^{2}$ can be topologically knotted (see Problem 1.102 (A/B) of Kirby's list [4]). In this note we construct knotted proper holomorphic embeddings of the unit disc in $\mathbb{C}^{2}$.

Theorem 1. There exist topologically knotted proper holomorphic embeddings of the unit disc in $\mathbb{C}^{2}$.

Since the image of a holomorphically embedded disc in $\mathbb{C}^{2}$ is a minimal surface, we obtain the following corollary, which solves Problem 1.102 (C) of Kirby's list.

Corollary 1. There exists a proper embedding $f: \mathbb{R}^{2} \rightarrow \mathbb{R}^{4}$ whose image is a topologically knotted complete minimal surface.

Our construction is based on the existence of locally well-behaved Fatou-Bieberbach domains and on the existence of knotted holomorphic discs in the 4-ball. A FatouBieberbach domain in $\mathbb{C}^{2}$ is an open subset of $\mathbb{C}^{2}$ which is biholomorphically equivalent to $\mathbb{C}^{2}$. Fatou-Bieberbach domains tend to have wild shapes; the first Fatou-Bieberbach domain with smooth boundary was constructed by Stensønes [10]. In [3], Globevnik constructed Fatou-Bieberbach domains $\Omega \subset \mathbb{C}^{2}$ whose intersections with $\mathbb{C} \times \Delta$ are arbitrarily small $\mathscr{C}^{1}$-perturbations of $\Delta \times \Delta$. Here $\Delta$ stands for the open unit disc in $\mathbb{C}$. These domains are well-adapted for constructing knotted holomorphic discs in $\mathbb{C}^{2}$. Indeed, all we need is a knotted proper holomorphic embedding of the closed unit disc $\varphi: \bar{\Delta} \rightarrow \bar{\Delta} \times \bar{\Delta}$ that maps $\partial \bar{\Delta}$ 
to $\partial \bar{\Delta} \times \Delta$. Composing the restriction of this embedding $\left.\varphi\right|_{\Delta}: \Delta \rightarrow \Delta \times \Delta \subset \Omega$ with a biholomorphism $h: \Omega \rightarrow \mathbb{C}^{2}$ yields a knotted proper holomorphic embedding of $\Delta$ in $\mathbb{C}^{2}$. The existence of knotted proper holomorphic embeddings $\varphi: \bar{\Delta} \rightarrow \bar{\Delta} \times \bar{\Delta}$ is easily established by using the theory of complex algebraic curves in $\mathbb{C}^{2}$.

We describe the relevant features of the Fatou-Bieberbach domains needed for our purpose in Section 2. The proof of Theorem 1 is completed in Section 3, where we give an explicit example of a knotted holomorphic disc in $\bar{\Delta} \times \bar{\Delta}$.

\section{Fatou-Bieberbach domains}

Fatou-Bieberbach domains in $\mathbb{C}^{2}$ are usually described by certain infinite processes, for example as domains of convergence of maps defined by sequences of automorphisms. This technique was used by Globevnik to construct Fatou-Bieberbach domains with controlled shape inside $\mathbb{C} \times \Delta$.

Theorem 2 (Globevnik [3]). Let $Q \subset \mathbb{C}$ be a bounded open set with boundary of class $\mathscr{C}^{1}$ whose complement is connected. Let $0<R<\infty$ be such that $\bar{Q} \subset R \Delta$. There are a domain $\Omega \subset \mathbb{C}^{2}$ and a volume-preserving biholomorphic map from $\Omega$ onto $\mathbb{C}^{2}$ such that:

(i) $\Omega \subset\left\{(z, w) \in \mathbb{C}^{2}:|z|<\max \{R,|w|\}\right\}$.

(ii) $\Omega \cap R(\Delta \times \Delta)$ is an arbitrarily small $\mathscr{C}^{1}$-perturbation of $Q \times R \Delta$.

The assumptions of Theorem 2 are verified when $Q$ is an open disc with smooth boundary (and $R>0$ large enough). This is the version we will need. As mentioned in the introduction, we will insert knotted discs in $\Delta \times \Delta$ with boundary in $\partial \bar{\Delta} \times \Delta$. It is a priori not clear whether such discs stay knotted in the larger domain $\Omega$. The following lemma allows us to control knottedness on the level of the fundamental group.

Lemma 1. Let $\Omega \subset \mathbb{C}^{2}$ be an open domain homeomorphic to $\mathbb{C}^{2}$ with

$$
\Omega \cap \mathbb{C} \times \Delta=\Delta \times \Delta,
$$

and let $X \subset \Delta \times \Delta$ be a subset with $\bar{X} \subset \bar{\Delta} \times \Delta$. The inclusion $i:(\Delta \times \Delta) \backslash X \rightarrow \Omega \backslash X$ induces an injective map $i_{*}: \pi_{1}((\Delta \times \Delta) \backslash X) \rightarrow \pi_{1}(\Omega \backslash X)$ (suppressing base points).

Remark. At this point it does not matter whether $\Omega \cap \mathbb{C} \times \Delta$ is precisely $\Delta \times \Delta$ or a small $\mathscr{C}^{1}$-perturbation of it. Further, the corresponding statement stays true if the intersection $\Omega \cap(\mathbb{C} \times \Delta)$ is a small $\mathscr{C}^{1}$-perturbation of $D \times \Delta$, where $D \subset \mathbb{C}$ is any embedded disc with smooth boundary.

Proof. Choose $\varepsilon>0$ such that $X \subset \Delta \times(1-\varepsilon) \Delta$. Setting

$$
U:=(\Delta \times \Delta) \backslash X
$$

and

$$
V:=\Omega \backslash(\Delta \times \overline{(1-\varepsilon) \Delta}),
$$


we have $\Omega \backslash X=U \cup V$. The theorem of Seifert-van Kampen tells us that $\pi_{1}(\Omega \backslash X)$ is the free product of $\pi_{1}(U)$ and $\pi_{1}(V)$ amalgamated over $\pi_{1}(U \cap V)$. The latter is isomorphic to $\mathbb{Z}$, since

$$
U \cap V=\Delta \times(\Delta \backslash \overline{(1-\varepsilon) \Delta})
$$

is homotopy equivalent to a circle. Let $\gamma: S^{1} \rightarrow U \cap V$ be a loop that generates $\pi_{1}(U \cap V)$. If the induced map $i_{*}: \pi_{1}((\Delta \times \Delta) \backslash X) \rightarrow \pi_{1}(\Omega \backslash X)$ were not injective, then a certain nonzero multiple of $[\gamma] \in \pi_{1}(V)$ would have to vanish. This is impossible since the inclusion $j: V \rightarrow \mathbb{C} \times \mathbb{C}^{*}$ maps $\gamma$ onto a generator of $\pi_{1}\left(\mathbb{C} \times \mathbb{C}^{*}\right) \cong \mathbb{Z}$.

\section{Complex plane curves}

A complex plane curve is the zero level $V_{f}=\left\{(z, w) \in \mathbb{C}^{2}: f(z, w)=0\right\} \subset \mathbb{C}^{2}$ of a non-constant polynomial $f(z, w) \in \mathbb{C}[z, w]$. Complex plane curves form a rich source of examples in algebraic geometry and topology. For example, the intersection of a complex plane curve $V_{f}$ with the boundary of a small ball centred at an isolated singularity of $f$ forms a link which is often called algebraic. The class of algebraic links has been generalized by Rudolph to the larger class of quasipositive links [7]. Using the theory of quasipositive links, it is easy to construct knotted proper holomorphic embedded discs in $\Delta \times \Delta$. In the following, we will give a short description of Rudolph's theory; more details are contained in [7] and [8].

Let $f(z, w)=f_{0}(z) w^{n}+f_{1}(z) w^{n-1}+\cdots+f_{n}(z) \in \mathbb{C}[z, w]$ be a non-constant polynomial, $f_{0}(z) \neq 0$. Under some generic conditions on $f$, the set $B$ of complex numbers $z$ such that the equation $f(z, w)=0$ has strictly less than $n$ solutions $w$, is finite. Let $\gamma \subset \mathbb{C} \backslash B$ be a smooth simple closed curve. The intersection $L=V_{f} \cap \gamma \times \mathbb{C}$ is a smooth closed 1dimensional manifold, i.e. a link, in the solid torus $\gamma \times \mathbb{C}$. More precisely, the link $L$ is an $n$-stranded braid in $\gamma \times \mathbb{C}$, which becomes a link in $S^{3}$ via a standard embedding of $\gamma \times \mathbb{C}$ in $S^{3}$. Links that arise in this way are called quasipositive.

If $D \subset \mathbb{C}$ is the closed disc bounded by $\gamma$, then the intersection $X=V_{f} \cap D \times \mathbb{C}$ is a piece of complex plane curve bounded by the link $L$. Choosing the polynomial $f$ and the curve $\gamma$ appropriately, it is possible to arrange $X$ to be an embedded disc with a non-trivial quasipositive knot as boundary (see [7], Example 3.2). Assuming that $X$ is compact, there exists $R>0$, such that $X \subset D \times R \Delta$. In order to establish Theorem 1, it remains to find an example where $X$ is knotted in $D \times R \Delta$, more precisely

$$
\pi_{1}(D \times R \Delta \backslash X) \neq \mathbb{Z} .
$$

In view of Lemma 1, this will then give rise to a knotted proper holomorphic embedding of the unit disc in $\Omega$, hence in $\mathbb{C}^{2}$.

Remark. Here we choose $\Omega \subset \mathbb{C}^{2}$ to be a Fatou-Bieberbach domain whose intersection $\Omega \cap \mathbb{C} \times R \Delta$ is a small $\mathscr{C}^{1}$-perturbation of $D \times R \Delta$. If this perturbation is small enough, then the intersection $\tilde{X}=V_{f} \cap \mathbb{C} \times R \Delta \cap \Omega$ is still a proper holomorphic embedded disc, as knotted as $X$ in $D \times R \Delta$. 
We conclude this section with a concrete example, in fact Rudolph's Example 3.2 of [7]. Let

$$
f(z, w)=w^{3}-3 w+2 z^{4}
$$

The complex plane curve $V_{f}$ is easily seen to be non-singular. The equation $f\left(z_{0}, w\right)=0$ fails to have three distinct solutions $w$, if and only if the two equations $f\left(z_{0}, w\right)=0$ and $\frac{\partial}{\partial w} f\left(z_{0}, w\right)=3 w^{2}-3=0$ have a simultaneous solution. This happens precisely when $z_{0}^{8}=1$. Thus the set $B$ consists of the 8 th roots of unity. For $z \in \mathbb{C} \backslash B$, the equation $f(z, w)=0$ has three distinct solutions $w_{1}, w_{2}, w_{3} \in \mathbb{C}$, which we index by increasing real parts. Further, it will be convenient to determine the set $B_{+}$of complex numbers $z$ such that the equation $f(z, w)=0$ has two distinct solutions $w$ with coinciding real parts. In our example, $B_{+}$consists of 8 rays emanating from the points of $B$, as shown in Figure 1 . These rays carry labels 1 or 2 , depending on whether the real parts of $w_{1}$ and $w_{2}$ or those of $w_{2}$ and $w_{3}$ coincide. There is a way of orienting the rays that corresponds to choosing positive standard generators of the braid group. Figure 1 also indicates a curve $\gamma$ that bounds a disc $D$. We claim that the piece of complex plane curve $X=V_{f} \cap D \times \mathbb{C}$ is a disc with $\pi_{1}((D \times \mathbb{C}) \backslash X) \nRightarrow \mathbb{Z}$.

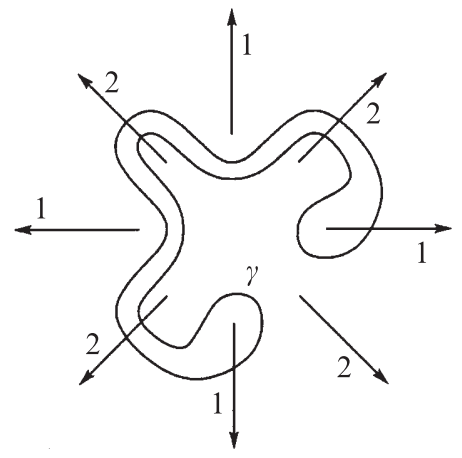

Figure 1

First, we observe that if we 'cut off' the two ends of the disc $D$ of Figure 1, we obtain another disc $D^{\prime}$ disjoint from $B$. The intersection $V_{f} \cap D^{\prime} \times \mathbb{C}$ is therefore a disjoint union of three discs. Adding the ends to $D^{\prime}$ again gives rise to two identifications along the boundaries of these discs. It is easy to see that these identifications result in a single disc whose boundary $L=V_{f} \cap(\gamma \times \mathbb{C})$ is knotted (actually $L$ is the quasipositive ribbon knot $8_{20}$ ). However, the mere fact that $L$ is knotted does not imply that the fundamental group of $(D \times \mathbb{C}) \backslash X$ is not isomorphic to $\mathbb{Z}$ (see the last paragraph of [2]). We will describe $\pi_{1}((D \times \mathbb{C}) \backslash X)$ explicitly, in terms of generators and relations. Hereby we will use Orevkov's method for presenting the fundamental group of the complement of complex plane curves in $\mathbb{C}^{2}$ [5]. To every connected component of $\mathbb{C} \backslash B_{+}$(in our case $D \backslash B_{+}$), we assign $n$ (in our case 3 ) generators corresponding to meridians of the $n$ discs lying over that component. Every oriented edge of the graph $B_{+}$gives rise to $n$ relations between the generators of the two (not necessarily distinct) components adjacent to that edge. All these relations are of Wirtinger-type (see [5], Lemma 3.1). 
Let us denote the four connected components of $D \backslash B_{+}$by $U_{1}, U_{2}, U_{3}, U_{4}$, starting at the bottom left and going clockwise around $D$. To every region $U_{j}$ correspond three generators $\alpha_{i j}=\alpha_{i}\left(U_{j}\right), 1 \leqq i \leqq 3$. The relations among these generators read as follows:

1. edge: $\quad \alpha_{11}=\alpha_{21}$.

2. edge: $\alpha_{11}=\alpha_{12}, \alpha_{31}=\alpha_{22}, \alpha_{21}=\alpha_{31} \alpha_{32} \alpha_{31}^{-1}$.

3. edge: $\alpha_{12}=\alpha_{13}, \alpha_{32}=\alpha_{23}, \alpha_{22}=\alpha_{32} \alpha_{33} \alpha_{32}^{-1}$.

4. edge: $\alpha_{13}=\alpha_{14}, \alpha_{33}=\alpha_{24}, \alpha_{23}=\alpha_{33} \alpha_{34} \alpha_{33}^{-1}$.

5. edge: $\alpha_{14}=\alpha_{24}$.

From this it is easy to verify that the assignment $\alpha_{11} \mapsto(23), \alpha_{31} \mapsto(12)$ defines a surjective homomorphism $\varphi: \pi_{1}((D \times \mathbb{C}) \backslash X) \rightarrow S_{3}$, whence $\pi_{1}((D \times \mathbb{C}) \backslash X)$ is not a cyclic group.

\section{References}

[1] S. S. Abhyankar and T. T. Moh, Embeddings of the line in the plane, J. reine angew. Math. 276 (1975), 148 166.

[2] S. Friedl and P. Teichner, New topologically slice knots, Geom. Topol. 9 (2005), 2129-2158.

[3] J. Globevnik, On Fatou-Bieberbach domains, Math. Z. 229 (1998), no. 1, 91-106.

[4] R. Kirby, Problems in Low-Dimensional Topology, www.math.berkeley.edu/ kirby/.

[5] S. Yu. Orevkov, The fundamental group of the complement of a plane algebraic curve (Russian), Mat. Sbornik 137 (179) (1988), no. 2, 260-270; English version: http://picard.ups-tlse.fr/ orevkov/.

[6] L. Rudolph, Embeddings of the line in the plane, J. reine angew. Math. 337 (1982), 113-118.

[7] L. Rudolph, Algebraic functions and closed braids, Topology 22 (1983), no. 2, 191-202.

[8] L. Rudolph, Knot theory of complex plane curves, Handbook of knot theory, Elsevier B. V., Amsterdam (2005), 349-427.

[9] A. R. Shastri, Polynomial representations of knots, Tohoku Math. J. (2) 44 (1992), no. 1, 11-17.

[10] B. Stensones, Fatou-Bieberbach domains with $C^{\infty}$-smooth boundary, Ann. Math. (2) 145 (1997), no. 2, 365-377.

ETH Zürich, Rämistrasse 101, 8092 Zürich, Switzerland e-mail: sebastian.baader@math.ethz.ch

Universität Bern, Sidlerstrasse 5, 3012 Bern, Switzerland e-mail: Frank.Kutzschebauch@math.unibe.ch

Universitetet I Oslo, Postboks 1053 Blindern, 0316 Oslo, Norway

e-mail: erlendfw@math.uio.no

Eingegangen 29. August 2008 